\title{
Prevalencia de anemia en gestantes de una zona sur andina de Ecuador considerando características prenatales
}

\section{Prevalence of anemia in pregnant women from a south Andean zone of Ecuador according to prenatal characteristics}

\author{
Susana Heredia Aguirre. ${ }^{1}$, Freddy Cuvi Guamán. ${ }^{2} \&$ Patricio Yánez Moretta. ${ }^{3}$ \\ Recibido: 15-01-2020 / Revisado: 20-03-2020 /Aceptado: 14-04-2020/ Publicado: 05-06-2020 \\ DOI: $\underline{\text { https://doi.org/10.33262/anatomiadigital.v3i2.1..1251 }}$
}

\begin{abstract}
Iron deficiency in pregnant women is relatively common, so a proper iron-rich diet is essential, in addition to supplement intake in order to avoid future complications. The objective of this research was to determine the prevalence of anemia in pregnant adult women in the southern Andean area of Ecuador, according to their prenatal characteristics. For this, anthropometric and biochemical data information of 164 mothers was recorded, with their consent, keeping their anonymity and considering the Helsinki criteria. Positive correlations were determined between two prenatal variables (age of the mother and her Body Mass Index) and her hemoglobin levels in the first trimester of pregnancy and in the postpartum period; part of the group of pregnant mothers
\end{abstract}

\begin{abstract}
Resumen.
La deficiencia de hierro en mujeres gestantes es relativamente común, por lo que es indispensable una correcta alimentación rica en hierro además de la ingesta de suplementos con la finalidad de evitar futuras complicaciones. El objetivo de la presente investigación fue el de determinar la prevalencia de anemia en mujeres adultas gestantes de la zona sur andina de Ecuador, según sus características prenatales. Para ello, se registró información de datos antropométricos y bioquímicos de 164 madres, bajo su consentimiento, guardando su anonimidad y considerando los criterios de Helsinki. Se determinaron correlaciones positivas entre dos variables prenatales (Edad de la madre y su Índice de Masa Corporal) y
\end{abstract}

\footnotetext{
1 Escuela Superior Politécnica de Chimborazo, Escuela de Nutrición y Dietética. Riobamba Ecuador. sisabelha@yahoo.com

2 Escuela Superior Politécnica de Chimborazo, Escuela de Nutrición y Dietética. Riobamba, Ecuador. cuviguaman@hotmail.com

${ }^{3}$ Universidad Internacional del Ecuador, Escuelas de Gestión Ambiental y de Turismo, Quito-Ecuador. Autor corresponsal: ayanez@uide.edu.ec; apyanez@hotmail.com
} 
suffered anemia during the first three months of pregnancy as well as in the postpartum period.

Key words: Pregnant mothers, Southern Ecuador, Anemia, Hemoglobin level. sus niveles de hemoglobina en el primer trimestre de embarazo y en el posparto; una parte del grupo de madres gestantes sufrió anemia durante los tres primeros meses de embarazo, así como en el postparto.

Palabras Clave: Madres gestantes, Sur de Ecuador, Anemia, Nivel de hemoglobina.

\section{Introducción.}

La anemia típicamente suele ser considerada como el estado de una persona que cuenta con una cantidad menor a la normal de eritrocitos circulantes o con una concentración disminuida de hemoglobina en la sangre; puede afectar a diferentes grupos humanos, entre ellos al de mujeres gestantes.

Generalmente, se considera que la anemia se origina por una ingesta insuficiente de alimentos que contienen hierro (Pazmiño-Tandazo et al., 2019). Los grupos más afectados a nivel mundial son: niños, mujeres adolescentes en edad fértil, mujeres en estado fisiológico de embarazo (De Benoist et al., 2008; Montoya et al. 2012). Estados de anemia avanzada en este último grupo pueden desembocar en una mayor morbimortalidad materno-fetal (Anlaakuu \& Anto, 2017).

La Organización Mundial de la Salud estima que el 42\% de las madres en estado de gestación sufre de anemia en alguna etapa de su embarazo (Espitia \& Orozco, 2013).

Debido a ello, durante la etapa del embarazo resulta necesario incrementar el consumo de hierro como medida preventiva para evitar futuras complicaciones en el feto y/o en el recién nacido (Milman, 2012). En este estado fisiológico los requerimientos de hierro van de 0,8 $\mathrm{mg} / \mathrm{dl}$ hasta 4,4 mg/dl; esta suplementación de hierro debe ir acompañada con ácido fólico con el propósito de prevención y aseguramiento del bienestar materno-fetal (Manjarrés et al., 2012).

El embarazo es un estado fisiológico de mayor requerimiento de hierro para la placenta y el feto, por lo que es indispensable un aumento razonable de este mineral en la alimentación, a pesar de ello durante la gestación pudiera ocurrir una disminución en la concentración de hemoglobina $(\mathrm{Hb})$, misma que se hace más evidente en el segundo trimestre del embarazo (Picciano, 2003).

La Organización Mundial para la Salud sugiere reducir el punto de corte para definir anemia a un valor de $\mathrm{Hb}$ de $11 \mathrm{~g} / \mathrm{dl}$ en la gestante, cuando en la no gestante es de $12 \mathrm{~g} / \mathrm{dl}$. Estos 
cambios en las concentraciones de hemoglobina en el segundo y tercer trimestre determinan que la prevalencia de anemia sea más alta en el segundo trimestre y disminuya al final del tercer trimestre (Tunkyi \& Moodley, 2018).

En Ecuador, el Ministerio de Salud Pública establece que el 46,9\% de madres gestantes llegan a presentar anemia (MSP, 2014). En este estado suele reportarse que las mujeres diagnosticadas con anemia tienen una mayor incidencia de infecciones urinarias, parto prematuro, amenazas de aborto, hemorragia obstétrica, recién nacidos con bajo peso, así como mayor número de ingresos a las unidades de cuidados intensivos (Iglesias et al., 2009).

En países cercanos como Perú, el protocolo dado por el Ministerio de Salud es suplementar hierro a todas las mujeres gestantes, anémicas o no (Gonzales \& Olavegoya, 2019).

Con estas consideraciones, se planteó el desarrollo de la presente investigación buscando conocer la prevalencia de anemia en mujeres gestantes y la asociación de ésta con algunas características prenatales en una zona andina del sur de Ecuador.

\section{Materiales y Métodos}

En este estudio participaron 164 mujeres embarazadas que se atendían en el Centro de Salud de Nabón (provincia de Azuay) a lo largo de 2017; los datos de estas mujeres se registraron bajo su consentimiento, guardando su anonimidad y considerando los principios éticos de la Declaración de Helsinki (WMA, 2017).

Hacia la semana 13 del embarazo se registraron sus datos antropométricos: talla y peso, utilizando una balanza digital con tallímetro y transmisión inalámbrica, marca Seca 703; con una capacidad de carga de $300 \mathrm{~kg}$, plataforma grande y plana y un rango de medición del tallímetro de 61-198 cm.

Para la toma de muestras para exámenes de laboratorio (hemoglobina) las madres gestantes debían estar en ayunas, la toma de muestras y su análisis se efectuó en la misma semana de embarazo y los realizó el personal de laboratorio del Centro de Salud.

\section{Resultados}

En la Tabla 1 se presentan los datos más relevantes para algunas características de las mujeres embarazadas, tales como la edad (que fluctuó entre 16 a 42 años), con una distribución ligeramente asimétrica, ya que el promedio fue mayor que la mediana.

La talla varió entre 1,35 m a 1,74 m; con una distribución más bien simétrica ya que el valor del promedio fue similar al de la mediana. 
Tabla 1. Características prenatales de las mujeres gestantes evaluadas, determinadas hacia la semana 13 del embarazo

\begin{tabular}{ccccccc}
\hline $\begin{array}{c}\text { Caracterí- } \\
\text { sticas }\end{array}$ & $\begin{array}{c}\text { Tamaño } \\
\text { de la } \\
\text { muestra }\end{array}$ & Mínimo & $\begin{array}{c}\text { Median } \\
\text { a }\end{array}$ & Máximo & $\begin{array}{c}\text { Prome- } \\
\text { dio }\end{array}$ & $\begin{array}{c}\text { Desvia- } \\
\text { ción } \\
\text { estándar }\end{array}$ \\
\hline Edad (años) & 164 & 16 & 23 & 42 & 24,58 & 6,13 \\
$\begin{array}{c}\text { Talla (metros) } \\
\text { IMC pregesta- } \\
\text { cional }\end{array}$ & 164 & 1,35 & 1,51 & 1,74 & 1,51 & 0,06 \\
\hline
\end{tabular}

Elaborado por : Grupo de investigación.

Para expresar el estado nutricional hacia el tercer mes del embarazo se calculó el Índice de Masa Corporal, encontrándose un IMC mínimo de $17,31 \mathrm{~kg} / \mathrm{m}^{2}$ y máximo de $34,67 \mathrm{~kg} / \mathrm{m}^{2}$, con un promedio de $24,48 \mathrm{~kg} / \mathrm{m}^{2}$. La distribución fue ligeramente asimétrica, ya que el valor del promedio fue mayor que el de la mediana (Tabla 1).

La ganancia de peso durante el embarazo fue clasificada considerando el estado nutricional al finalizar el tercer trimestre del embarazo. El 32,21 \% presentó una baja ganancia de peso; el 58,18\%, una ganancia normal de peso; y, el 9,69 \%, una ganancia de peso mayor a la recomendada.

En este sentido, cabe mencionar que es importante observar si existen o no niveles de obesidad en las mujeres gestantes, dado que en algunos estudios como el de Gonzales (2011) se menciona que las mujeres obesas tienen menos probabilidades de tener una buena hemoconcentración.

También es importante señalar que una gestante con un diagnóstico de sobrepeso u obesidad debería tener en general una ganancia menor de peso (Khoigani et al., 2012).

Con respecto a los valores de prevalencia de anemia, se presentan en la Tabla 2.

Al final del primer trimestre del embarazo solo el 12,1\% presentó algún nivel de anemia (anemia leve); mientras que en el post parto el 32,9 \% presentó anemia de leve a severa (Tabla $2)$. 
Tabla 2. Prevalencia de anemia en mujeres evaluadas, al final del primer trimestre de gestación y en post parto

\begin{tabular}{lcccc}
\hline HEMOGLOBINA & \multicolumn{2}{c}{ PRIMER TRIMESTRE } & \multicolumn{2}{c}{ POST PARTO } \\
& Frecuencia & Porcentaje & Frecuencia & Porcentaje \\
\hline Normal & 144 & 87,87 & 110 & 67,07 \\
Anemia leve & 20 & 12,13 & 31 & 18,90 \\
Anemia moderada & 0 & 0,00 & 22 & 13,42 \\
Anemia severa & 0 & 0,00 & 1 & 0,61 \\
\hline TOTAL & $\mathbf{1 6 4}$ & $\mathbf{1 0 0 , 0 0}$ & $\mathbf{1 6 4}$ & $\mathbf{1 0 0 , 0 0}$ \\
\hline
\end{tabular}

Elaborado por : Grupo de investigación.

Las mujeres que presentaron anemia mostraron a la vez poco consumo de ácido fólico y vitamina B12. Se debe recordar que el folato se caracteriza por tener funciones importantes en todos los procesos celulares, biosíntesis de ácidos nucleicos, biogénesis de grupos metilos y metabolismo de los aminoácidos. La detección oportuna de esta deficiencia podría ayudar a reducir el riesgo de defectos del tubo neural. Es importante mencionar que el exceso de folatos también puede causar daño al bebé relacionados con la ocurrencia de autismo, por lo que en general se sugiere no exceder su consumo y cumplir las recomendaciones (Lassi et al., 2013).

Lo mencionado evidencia la importancia de detectar casos como estos y tratarlos como corresponda para lograr un embarazo adecuado, sin complicaciones ni para el bebé ni para la madre.

En la Tabla 3, se observan los niveles de correlación detectados entre las variables: Edad de la Madre e IMC con los valores de hemoglobina tomados en dos momentos de interés.

Tabla 3. Correlación entre variables prenatales y valores de hemoglobina

\begin{tabular}{llcc}
\hline \multicolumn{1}{c}{$\begin{array}{c}\text { Variable } \\
\text { prenatal }\end{array}$} & \multicolumn{1}{c}{ Hemoglobina } & $\begin{array}{c}\text { Valor de Correlación } \\
(\mathbf{r})\end{array}$ & $\mathbf{p}$ \\
\hline \multirow{2}{*}{ Edad de la Madre } & $\begin{array}{l}\text { Hemoglobina 1er. } \\
\text { trimestre }\end{array}$ & 0,19 & 0,02 \\
& Hemoglobina postparto & 0,26 & 0 \\
\hline $\begin{array}{l}\text { IMC 1er. } \\
\text { Trimestre }\end{array}$ & Hemoglobina postparto & 0,2 & 0,01 \\
\hline
\end{tabular}

Elaborado por : Grupo de investigación. 
En general, las tres relaciones evaluadas muestran valores de correlación (r) bajos, pero significativos. Sugiriendo una relación consistente entre ambas variables, apoyada por un tamaño muestral importante (n > 100) (Yánez, 2005).

La relación entre edad de la madre y el nivel de hemoglobina postparto en relación con la edad $(r=0,26)$ demuestra que entre más edad tenga la mujer mejores niveles de hemoglobina contiene en comparación a aquellas mujeres adolescentes que terminan el embarazo con niveles bajos de hemoglobina.

La correlación entre los valores de IMC 1er. Trimestre (pregestacional) y la Hemoglobina postparto $(r=0,20)$ permite determinar que los niveles de hemoglobina se incrementan suave pero consistentemente en aquellas mujeres con IMC superior a lo normal (sobrepeso u obesidad). Esto es considerado un factor de riesgo, ya que se ha demostrado que si se tiene un IMC elevado existe mayor riesgo de sufrir anemia posparto (Gonzales, 2011).

Además, otro hallazgo importante en el presente estudio es que las mujeres embarazadas ganaron adecuadamente el peso requerido durante el período de gestación.

\section{Discusión}

Considerando que se define como anemia a la concentración de Hemoglobina $<11 \mathrm{~g} / \mathrm{dL}$ de sangre o un hematocrito $<33 \%$ en los tres primeros meses de embarazo o $<32 \%$ en el segundo trimestre (WHO, 2011). un valor de hemoglobina menor a $11 \mathrm{~g} / \mathrm{dL}$ especialmente al final del período de gestación se debería considerar como un indicativo de deficiencia de hierro (Azulay et al., 2015).

La deficiencia de hierro se asocia a un riesgo tres veces mayor de bajo peso al nacer y dos veces mayor de parto pre-término, retraso en el crecimiento intrauterino, alteración del desarrollo placentario y aumento del riesgo de pérdidas fetales (San Gil et al., 2013). En el presente estudio, el $12,13 \%$ de la población fue diagnosticada con anemia leve durante el primer trimestre de embarazo, y no se detectó ningún porcentaje con anemia moderada ni severa; esta anemia leve no suele causar complicaciones graves y generalmente se encuentra relacionada con algunas deficiencias en el consumo de micronutrientes y algunas enfermedades en curso de la madre.

En estudios realizados en zonas similares de Perú se indica que la prevalencia de anemia aumenta conforme a la edad de la madre, pero tiende a ser más común el encontrarse con cuadros de anemia leve (Ministerio de Salud, 2012).

En otro estudio en México se evidenció que los principales factores que se asocian a la aparición de anemia en mujeres gestantes es un Índice de Masa Corporal pregestacional bajo, 
tres o más partos, alimentación deficiente, ausencia de suplementación, falta de educación nutricional (Barba \& Cabanillas, 2007).

Es importante realizar un control en mujeres embarazadas para descartar anemia con el propósito de disminuir el riesgo de parto pretérmino; según Wang et al. (2018) el diagnóstico de anemia materna leve en el segundo y tercer trimestre no tiene relación con el riesgo de parto pretérmino ya que estos casos se mezclan con aquellos valores que presentan una hemoglobina baja $<11 \mathrm{~g} / \mathrm{dL}$, debido a hemodilución.

Asimismo, Kozuki et al. (2012) mencionan que si la madre es diagnosticada con anemia moderada y/o severa en los primeros meses de embarazo, el bebé tiene mayor riesgo de nacer pequeño. Otros estudios concuerdan con esta aseveración y a la vez mencionan que si se detecta anemia severa, ello constituye un factor de riesgo para morbilidad y mortalidad tanto materna como neonatal (Wylie, 2019; Serdula et al., 2018).

Asimismo, otros autores también indican que la anemia leve no es necesariamente una evidencia patológica para la mujer embarazada, relacionándose más bien a la falta de respuesta de suplementación (Viteri et al., 2012). Esto puede corregirse ya que al incrementar la administración diaria con hierro (60 a $120 \mathrm{mg}$ ), aumenta el estrés oxidativo produciendo un incremento en la concentración de hemoglobina en mujeres embarazadas no anémicas (Jirakittidul et al., 2018).

Igualmente, cabe mencionar que la intervención en mujeres embarazadas no anémicas, con la suplementación de hierro antes de los cuatro meses aproximadamente de gestación se asocia a un mayor riesgo de desarrollar hipertensión arterial después de la semana 20 de embarazo. Con este hallazgo se puede mencionar que una suplementación innecesaria puede exponer a un riesgo a las mujeres embarazadas que no ameriten tal suplementación (Wainstock et al., 2019).

Asimismo, cabe indicar que algunas vitaminas y minerales coadyuvan a la función del hierro en la eritropoyesis, como por ejemplo: vitamina A, cobre, manganeso y zinc (WesslingResnick, 2017).

Una forma de evitar esta posible deficiencia de hierro es con una adecuada alimentación, educación nutricional y suplementación cuando sea necesaria (Bonete et al., 2016; Cuvi, 2018; Heredia et al., 2016). En la mayoría de países, se utiliza la suplementación de hierro y ácido fólico para prevenir la anemia durante la etapa del embarazo, para lo cual se basan en los esquemas de suplementación con hierro durante toda o casi toda la etapa del embarazo, lo cual suele reducir la incidencia de partos prematuros y bajo peso al nacer (Neves et al., 2018). 


\section{Conclusiones.}

- En esta investigación se pudo observar que un porcentaje de las madres gestantes evaluadas sufrió anemia durante los tres primeros meses (anemia leve) como después del post parto (leve a moderada) y que su ocurrencia tuvo una relación inversa con la edad de la madre y su índice de masa corporal pregestacional.

- Se recomienda que toda mujer en estado de gestación debe asistir a los controles médicos, dentro de ellos acudir a un profesional Nutricionista para recibir una adecuada educación alimentaria nutricional y alimentarse con alimentos fuentes de hierro y ácido fólico, antes, durante y después de su etapa de gestación para evitar futuras complicaciones.

- Asimismo, resulta necesario realizar un correcto diagnóstico para la determinación de anemia (y su nivel) en la mujer gestante, ya que puede ésta ocurrir por deficiencia de hierro o procesos inflamatorios, a la vez es imprescindible evaluar otros marcadores de estado del hierro. Por ejemplo, se debería considerar el nivel del volumen plasmático en relación con la concentración de hemoglobina en aquellas mujeres que han sido consideradas con sobrepeso y obesidad.

\section{Referencias Bibliográficas}

Anlaakuu P, \& Anto, F. (2017). Anaemia in pregnancy and associated factors: a cross sectional study of antenatal attendants at the Sunyani Municipal Hospital, Ghana. BMC Research Notes, 10(1): 402.

Azulay, C., Pariente, G., Shoham-Vardi , I., \& Kessous , R. (2015). Maternal anemia during pregnancy and subsequent risk for cardiovascular disease. J Matern Fetal Neonatal Med, 28(15):1762-1765.

Barba-Oropeza, F., \& Cabanillas-Gurrola, J. (2007). Factores asociados a la anemia durante el embarazo en un grupo de gestantes mexicanas. Archivos de Medicina Familiar, 9(4), 170-175.

Bonete, M., Urquizo, C., Guevara, R., \& Yánez, P. (2016). Estudio de cuatro tubérculos y raíces tuberosas no tradicionales de la sierra centro de Ecuador y su potencial de uso en platos de autor. Qualitas, 12, 37-67.

Cuvi Guamán, F. J. (2018). Estado nutricional pregestacional y ganancia de peso materno durante la gestación y su relación con el peso, longitud al nacer, en el Centro de Salud de Nabón. Riobamba: Escuela Superior Politécnica de Chimborazo. Tesis de Maestría.

De Benoist, B., Mclean, E., Egli, I., \& Cogswell, M. (2008). WHO Global Database on Anemia. Ginebra: WHO.

Espitia, F., \& Orozco, L. (2013). Anemia en el embarazo, un problema de salud que puede prevenirse. Revista Médicas UIS, 26(3): 45-50.

Gonzales, G. (2011). Hemoglobin and testosterone: importance on high altitude acclimatization and adaptation. Rev Peru Med Exp Salud Publica, 28:92-100. 
Gonzales, G., \& Olavegoya, P. (2019). Fisiopatología de la anemia durante el embarazo: ¿anemia o hemodilución? Revista Peruana de Ginecología y Obstetricia, 65(4) 489502.

Heredia, S., Robalino, M., Hidalgo, M., Proaño, F., Antamba, E., \& Yánez, P. (2016). Caracterización del perfil lipídico, índice de masa corporal y nivel de glucosa en afiliados del Instituto Ecuatoriano de Seguridad Social (IESS) Riobamba, 2014, como parámetros indicadores de su estado de salud. Qualitas, 12, 124-134.

Huisman, A., \& Aarnoudse, J. (1986). Increased 2nd trimester hemoglobin concentration in pregnancies later complicated by hypertension and growth retardation. Early evidence of a reduced plasma volume. Acta Obstetricia et Gynecologica Scandinavica, 65(6): 605-608.

Iglesias-Benavides, J., Tamez-Garza, L., \& Reyes-Fernández, I. (2009). Anemia y embarazo, su relación con complicaciones maternas y prenatales. Medicina Universitaria, 11(43): 95-98.

Jirakittidul, P., Sirichotiyakul, S., Ruengorn, C., Techatraisak, K., \& Wiriyasirivaj, B. (2018). Effect of iron supplementation during early pregnancy on the development of gestational hypertension and pre-eclampsia.Archives of gynecology and obstetrics, 298(3), 545-550.

Keats, E., Haider, B., Tam, E., \& Bhutta, Z. (2019). Multiple-micronutrient supplementation for women during pregnancy. Cochrane Database of Systematic Reviews, 3.

Khoigani, M., Goli, S., \& Hasanzadeh, A. (2012). The relationship of hemoglobin and hematocrit in the first and second half of pregnancy with pregnancy outcome. Iranian Journal of Nursing and Midwifery Research, 17(1): S165.

Kozuki , N., Lee , A., \& Katz , J. (2012). Child Health Epidemiology Reference Group. Moderate to severe, but not mild, maternal anemia is associated with increased risk of small-for-gestational-age outcomes. J Nutr, 142(2):358-62.

Lassi, Z., Salam, R., Haider, B., \& Bhutta, Z. (2013). Folic acid supplementation during pregnancy for maternal health and pregnancy outcomes. Cochrane Database of Systematic Reviews, 3.

Manjarrés, L., Parra, B., Díaz, A., Restrepo, S., \& Mancilla, L. (2012). Ingesta de hierro y folatos durante el embarazo y su relación con indicadores bioquímicos maternos. Iatreia, 25(3):194-202.

Milman, N. (2012). Fisiopatología e impacto de la deficiencia de hierro y la anemia en las mujeres gestantes y en los recién nacidos/infantes. Revista Peruana de Ginecología y Obstetricia, 58(4), 293-312.

Ministerio de Salud. (2012). Anemia en gestantes del Perú y Provincias con comunidades nativas. Informe de la Dirección Ejecutiva de Vigilancia Alimentaria y Nutricional. Lima.

Ministerio de Salud Pública del Ecuador - MSP. (2014). Guías de Práctica Clínica. Disponible en: www.salud.gob.ec/guias-de-practica-clinica/ 
Montoya, C., Castelazo, E., Valerio, E., Velásquez, G., Nava, D., Escarcega, J., \& et.al. (2012). Opinión de un grupo de expertos en diagnóstico y tratamiento de la anemia en la mujer embarazada. Ginecol. Obstet. Mex., 80(9): 563-580.

Neves, P., Castro, M., Oliveira, C., Malta, M., Lourenço, B., \& Cardoso, M. (2018). Effect of vitamin A status during pregnancy on maternal anemia and newborn birth weight: results from a cohort study in the Western Brazilian Amazon. European Journal of Nutrition, 1-12.

Pazmiño-Tandazo, P., Heredia-Aguirre, S., \& Yánez-Moretta, P. (2019). Factores de riesgo relacionados con deficiencia nutricional en niños de una comunidad centro andina de Ecuador. Revista Científica "Conecta Libertad", 3(2), 13-23.

Picciano, M. (2003). Pregnancy and lactation: physiological adjustments, nutritional requirements and the role of dietary supplements. The Journal of Nutrition, 133(6):1997S-2002S.

San Gil, C., Villazán, C., \& Ortega, Y. (2013). Caracterización de la anemia durante el embarazo y algunos factores de riesgo asociados, en gestantes del municipio regla. Rev Cubana Med Gen Integr, 30(1):71-81.

Serdula, M., Zhou, Y., Li, H., Liu, J., \& Mei, Z. (2018). Prenatal iron containing supplements provided to Chinese women with no or mild anemia had no effect on hemoglobin concentration in post-partum women or their infants at 6 and 12 months of age. Eur J Clin Nutr, 73(11), 1473-1479.

Tunkyi, K., \& Moodley, J. (2018). Anemia and pregnancy outcomes: a longitudinal study. The Journal of Maternal-Fetal \& Neonatal Medicine, 31(19): 2594-2598.

Viteri, F., Casanueva, E., Tolentino, M., Díaz, J., \& Erazo, A. (2012). Antenatal iron supplements consumed daily produce oxidative stress in contrast to weekly supplementation in Mexican non-anemic women. Reprod Toxicol, 34, 125-132.

Wainstock, T., Walfisch, A., Sergienko, R., \& Sheiner, E. (2019). Maternal anemia and pediatric neurological morbidity in the offspring-Results from a population based cohort study. Early human development, 128, 15-20.

Wang, C., Lin, L., Su, R., Zhu, W., Wei, Y., Yan, J., \& Yang, H. (2018). Hemoglobin levels during the first trimester of pregnancy are associated with the risk of gestational diabetes mellitus, pre-eclampsia and preterm birth in Chinese women: a retrospective study. BMC Pregn Childbirth, 18(1):263.

Wessling-Resnick, M. (2017). Excess iron: considerations related to development and early growth. The American Journal of Clinical Nutrition, 106(6):1600S-5S.

WHO - WORLD Health Organization. (2011). Haemoglobin concentrations for the diagnosis of anaemia and assessment of severity. Disponible en: http://www.who.int/vmnis/indicators/haemoglobin/en/

World Medical Association - WMA. (2017). Declaration of Helsinki-ethical principles for medical research involving human subjects. Disponible en: wma.net/policies- 
post/wma-declaration-of-helsinki-ethical-principles-for-medical-research-involvinghuman-subjects/

Wylie, B. (2019). Severe maternal anaemia, a missed opportunity for prevention. BJOG. Disponible en: https://doi.org/10.1111/1471-0528.15585

Yánez, P. (2005). Biometría y Bioestadística fundamentales. Analizando la estructura numérica de la información en proyectos ecológicos. Quito. 130pp.

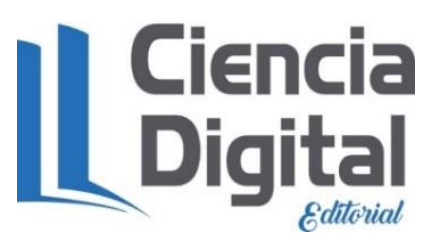




\section{PARA CITAR EL ARTÍCULO INDEXADO.}

Heredia Aguirre, S., Cuvi Guamán, F., \& Yánez Moretta, P. (2020). Prevalencia de anemia en gestantes de una zona sur andina de Ecuador considerando características prenatales. Anatomía Digital, 3(2.1.), 6-17. DOI: https://doi.org/10.33262/anatomiadigital.v3i2.1..1251

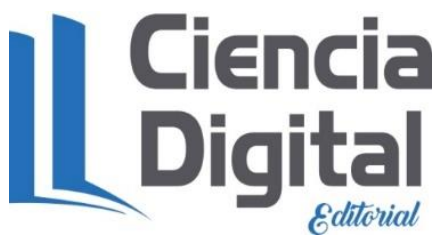

El artículo que se publica es de exclusiva responsabilidad de los autores y no necesariamente reflejan el pensamiento de la Revista Anatomía Digital.

El artículo queda en propiedad de la revista y, por tanto, su publicación parcial y/o total en otro medio tiene que ser autorizado por el director de la Revista Anatomía Digital.
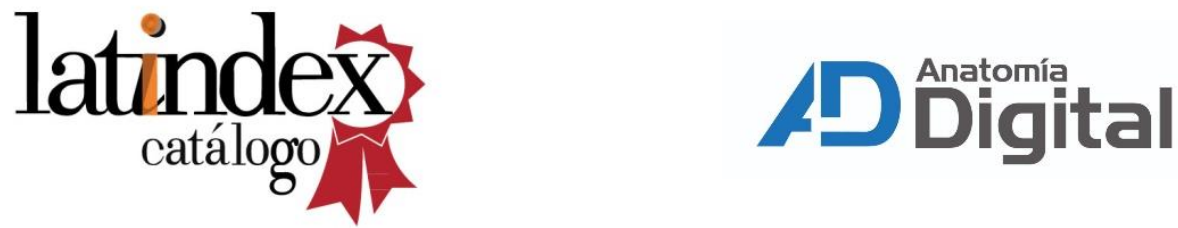